Copyright (C) 2013 IEEE. Personal use of this material is permitted. Permission from IEEE must be obtained for all other uses, in any current or future media, including reprinting/republishing this material for advertising or promotional purposes, creating new collective works, for resale or redistribution to servers or lists, or reuse of any copyrighted component of this work in other works. 


\title{
Autonomous Operation of Multiple Interconnected Microgrids with Self-Healing Capability
}

\author{
Farhad Shahnia ${ }^{1}$, Ruwan P.S. Chandrasena ${ }^{2}$, Sumedha Rajakaruna ${ }^{3}$ and Arindam Ghosh $^{4}$ \\ ${ }^{1,2,3}$ Centre of Smart Grid and Sustainable Power Systems, Electrical and Computer Engineering Department, \\ Curtin University, Perth, Australia \\ ${ }^{4}$ School of Electrical Engineering and Computer Science, Queensland University of Technology, Brisbane, Australia. \\ ${ }^{1}$ farhad.shahnia@curtin.edu.au, ${ }^{2}$ ruwan.chandrasena@curtin.edu.au, ${ }^{3}$ s.rajakaruna@curtin.edu.au, ${ }^{4}$ a.ghosh@qut.edu.au
}

\begin{abstract}
This paper shows how multiple interconnected microgrids can operate in autonomous mode in a self-healing medium voltage network. This is possible if based on network selfhealing capability, the neighbour microgrids are interconnected and a surplus generation capacity is available in some of the Distributed Energy Resources (DERs) of the interconnected microgrids. This will reduce or prevent load shedding within the microgrids with less generation capacity. Therefore, DERs in a microgrid are controlled such that they share the local load within that microgrid as well as the loads in other interconnected microgrids. Different control algorithms are proposed to manage the DERs at different operating conditions. On the other hand, a Distribution Static Compensator (DSTATCOM) is employed to regulate the voltage. The efficacy of the proposed power control, sharing and management among DERs in multiple interconnected microgrids is validated through extensive simulation studies using PSCAD/EMTDC.
\end{abstract}

Index Terms-Interconnected Microgrids, Self-Healing Network, Power Sharing, DSTATCOM.

\section{INTRODUCTION}

Smart Grid (SG) is a term referred to the improved status of existing electric networks into more reliable, efficient, sustainable and customer-interactive conditions by properly adding advanced metering, protection and communication infrastructures [1-2]. Among various anticipated smart features, self-healing is a key attribute in SGs. In self-healing, it is expected that the network can continuously detect, analyze and respond to faults and restore feeders with minimum human participation [3]. Therefore, in the case of a fault in the network, the normal operation could be restored in different feeder sections by properly isolating only the faulted subsections such that the amount of loads affected are minimized.

Another key aspect of smart grid technology is increasing the participation of Distributed Energy Resources (DER) [3]. Microgrid (MG) is a cluster of DERs and loads which can operate in grid-connected or autonomous modes [4]. In gridconnected mode, the DERs in a MG can be operating at their rated condition while in autonomous mode, if the DER generation capacity in the MG is more or equal to local load demand, they will be sharing the loads. However, if the DER generation capacity in the MG is less than the local load demand, load shedding has to be carried out in order to maintain the voltage and frequency in the MG [5]. In [6], the concept of distributed autonomous MGs was proposed in which the DERs can be controlled to share the power among neighbour MGs. In such a concept, if the DER generation capacity in a MG is less than the local load demand but there is available generation capacity by the DERs in the neighbour MGs, interconnecting MGs can reduce the load shedding. With the increased interest in SG and self-healing characteristics, there will be a possibility of interconnecting MGs in near future. This is the main research scope of this paper.

\section{SELF-HEALING}

Let us assume a radial Medium Voltage (MV) feeder with a single-automated switch installed somewhere in the middle of the feeder [7]. During a fault, the substation circuit breaker will first open and then reclose after a fixed time delay. If the fault is still present, the circuit breaker reopens again. The midpoint switch opens the circuit if it senses a voltage drop for more than 30 seconds. After a second fixed time delay, the substation circuit breaker closes the circuit again. Now, if the fault was on the downstream of the midpoint switch, the circuit breaker remains closed and the customers on the nonfaulty section are energized. Otherwise, if the fault is between the substation and the midpoint switch, the substation circuit breaker will reopen the feeder. This is the most common existing practice of feeder automation these days. However, by integration of DERs and increasing the number of switches along the feeder, the number of affected customers can be significantly reduced. This can be achieved by applying a fixed time delay between the switching operations or if the switches are equipped with communication capabilities among themselves using fibre optics or radio frequency.

Improvement in system reliability is driving the grids into smart grid concept with self-healing capability [2,8]. The feasibility study carried out in [9] concludes that integration of self-healing capabilities for the future smart grids is inevitable due to their high financial benefits to utilities by reducing the amount of energy not supplied and the number of affected customers.

For a self-healing network, the intelligent agents are required to adapt the system operation conditions. These agents are then utilized for analysing and maintaining system reliability in real-time. A framework, required to allow deployment of autonomous agents throughout an interconnected system, was proposed in [10-11]. Such a framework can be 
utilized to support a self-healing smart grid through monitoring and control. Some self-healing reconfiguration techniques were proposed in [12-14] to divide the network into isolated grids while minimizing the number of effected loads. Some utilities in US have already started implementing selfhealing projects [7]. It is to be reminded that following assumptions are required for the self-healing networks:

- Sufficient DER capability in MG

- Availability of communication interfaces among DERs, protection devices, circuit breakers and switches.

\section{NETWOK AND MICROGRID STRUCTURE}

Let us consider a MV feeder connected to two MGs as shown in Fig. 1. MG-1 has 3 DERs and 5 loads while MG-2 has 2 DERs and 4 loads, connected to Low Voltage (LV) feeders. A Distribution Static Compensator (DSTATCOM) is installed at the secondary side of the distribution transformer in each MG to regulate the voltage at its Point of Common Coupling (PCC). The loads are assumed as residential loads and all DERs are assumed to be converter-interfaced DERs.

During grid connected mode, all DERs will be operating in their rated conditions or based on the outcomes for economic analyses. In autonomous mode, when $\mathrm{CB}_{\mathrm{M} 1}$ and $\mathrm{CB}_{\mathrm{M} 2}$ are open, the DERs inside each MG will be sharing the loads within each MG separately. In autonomous mode, if the power demand in each MG is higher than the power generation capacity of the DERs in that MG, load shedding must be applied to some of the (non-critical) loads in that MG.

Now, let us assume that a fault occurs on the upstream of $\mathrm{CB}_{\mathrm{G}}$. Based on the network protection coordination, $\mathrm{CB}_{\mathrm{G}}$, $\mathrm{CB}_{\mathrm{M} 1}$ and $\mathrm{CB}_{\mathrm{M} 2}$ open under such conditions. Now, each $\mathrm{MG}$ can work in autonomous mode. However, let us assume a situation where the power generation in MG-2 is less than its power demand; while the power generation capacity in MG-1 is higher than its power demand. Now, let us assume the network has self- healing capability and $\mathrm{CB}_{\mathrm{M} 1}$ and $\mathrm{CB}_{\mathrm{M} 2}$ close the circuit while $\mathrm{CB}_{\mathrm{G}}$ remains open. In this way, the two MGs are now connected together. Hence, the DERs in MG-1 can share some of the loads in MG-2 and prevent/reduce load shedding in MG-2. This paper focuses on the power sharing and voltage regulation issues of the network in these situations. The protection devices, their coordination, communication and resynchronization of interconnecting MGs are beyond the scope of this paper.

\subsection{Power Sharing in Microgrid}

In this section, the power sharing method within the MG is discussed. For a converter-interfaced DER, with the structure shown in Fig. 2(a), the instantaneous active and reactive power flows from converter output to its $\mathrm{PCC}$ are

$$
\begin{aligned}
& p=\frac{\left|V_{T}\right| \times\left|V_{c f}\right|}{\omega L_{T}} \sin \left(\delta_{c f}-\delta_{T}\right) \\
& q=\frac{\left|V_{T}\right|}{\omega L_{T}}\left(\left|V_{c f}\right| \cos \left(\delta_{c f}-\delta_{T}\right)-\left|V_{T}\right|\right)
\end{aligned}
$$

where $V_{T}=\left|V_{T}\right| \angle \delta_{T}$ and $V_{c f}=\left|V_{c f}\right| \angle \delta_{c f}$ are respectively the RMS value of PCC voltage and AC filter capacitor voltage of

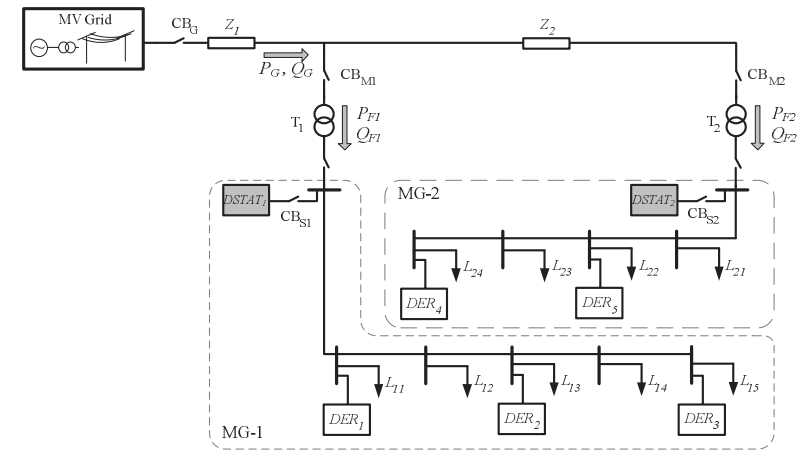

Fig. 1. Schematic diagram of the network under consideration.

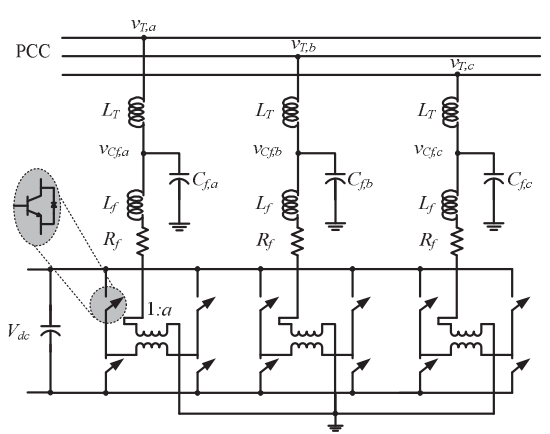

(a)

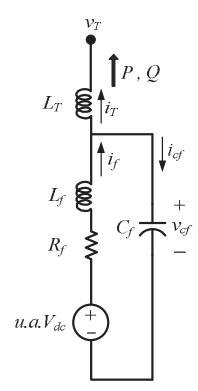

(b)
Fig. 2. (a) Schematic diagram of VSC for DERs and DSTATCOMs, (b) Single-phase equivalent circuit of VSC.

DER. $\omega L_{T}$ is the impedance of the coupling inductance between these two points which controls DER output power flow. Passing the instantaneous active power $(p)$ and reactive power $(q)$ through a low pass filter, the average active power $(P)$ and reactive power $(Q)$ can be calculated. In [15], it was proposed that decentralized power sharing among the DERs can be achieved, similar to conventional droop control, by changing the voltage magnitude and angle of DERs as

$\delta_{c f}=\delta_{\text {rated }}-m\left(P_{\text {rated }}-P\right)$

$V_{c f}=V_{\text {rated }}-n\left(Q_{\text {rated }}-Q\right)$

where $V_{\text {rated }}$ and $\delta_{\text {rated }}$ are respectively the rated voltage magnitude and angle of the DER when supplying the load with rated active and reactive power of $P_{\text {rated }}$ and $Q_{\text {rated }}$ while $m$ and $n$ are the droop coefficients.

The principle of decentralized power sharing in the MG is based on keeping the power output of DERs proportional to their ratings. This was mathematically simplified in [15] as

$$
\begin{array}{ll}
\frac{P_{1}}{P_{2}} \approx \frac{m_{2}}{m_{1}}=\frac{P_{1 \text { rated }}}{P_{2 \text { rated }}} \quad, \quad \frac{P_{1}}{P_{3}} \approx \frac{m_{3}}{m_{1}}=\frac{P_{1 \text { rated }}}{P_{3 \text { rated }}}, \quad, \ldots \\
\frac{Q_{1}}{Q_{2}} \approx \frac{n_{2}}{n_{1}}=\frac{Q_{1 \text { rated }}}{Q_{2 \text { rated }}} \quad, \quad \frac{Q_{1}}{Q_{3}} \approx \frac{n_{3}}{n_{1}}=\frac{Q_{1 \text { rated }}}{Q_{3 \text { rated }}} \quad, \ldots
\end{array}
$$

where the number suffixes show the number of each DER.

\subsection{DER Converter Structure}

The converter-interfaced DERs such as photovoltaic cells (PV), fuel cells and batteries are connected to the MG through voltage source converters (VSC) as shown in Fig. 1. All the DERs have a VSC structure consisted of three singlephase H-bridges, using IGBTs, as shown in Fig. 2(a). Each 
IGBT has proper parallel reverse diode and snubber circuits. The outputs of each $\mathrm{H}$-bridge are connected to a single-phase transformer, with $1: a$ ratio, and three transformers are starconnected. In this figure, the resistance $R_{f}$ represents the switching and transformer losses, while the inductance $L_{f}$ represents the leakage reactance of the transformers and the filter capacitor $C_{f}$ is connected to the output of the transformers to bypass the switching harmonics.

\subsection{DER Converter Control}

In this paper, the main concept of DER converter control is based on fixing the voltage magnitude and angle across $C_{f}$ as defined by the droop control from (2)-(3). This is achieved by appropriate switching of IGBTs in the converter. For this, let us consider the single-phase equivalent circuit of VSC as shown in Fig. 2(b). In this figure, $u \cdot a \cdot V_{d c}$ represents the converter output voltage, where $u$ is the switching function that can take on \pm 1 value depending on which pair of the IGBTs is turned on. The VSC is utilizing a closed-loop optimal robust controller based on state feedback to generate $u$. For this, two different state vectors can be assumed.

First, let us assume the state vector be defined as

$x=\left[\begin{array}{lll}v_{c f} & i_{c f} & i_{T}\end{array}\right]^{T}$

where $v$ and $i$ represent the instantaneous voltage and current, respectively. We refer to this as 3-parameter control mode. Now, the reference vector, $x_{r e f}$ is to be calculated for each state variable. For this, each DER will have

$v_{c f, r e f}=V \angle \delta, i_{c f, r e f}=\frac{v_{c f, r e f}}{j \omega C_{f}}, i_{T, r e f}=\frac{\sqrt{P^{2}+Q^{2}}}{V} \angle\left(\delta-\tan ^{-1} \frac{Q}{P}\right)$

On the other hand, the state vector can also be defined as $x=\left[\begin{array}{ll}v_{c f} & \tilde{i}_{f}\end{array}\right]^{T}$

where $\tilde{l}_{f}$ is the high frequencies of $i_{f}$. We refer to this as 2parameter control mode. In this mode, the reference vector, $x_{r e f}$ is

$v_{c f, r e f}=V \angle \delta, \tilde{i}_{f, r e f}=0$

For DER control in this paper, the 2-parameter control mode is used during grid-connected mode of MGs and 3parameter control mode is used during autonomous mode.

For both of the above mentioned modes, from the circuit of Fig. 2(b), system state space description can be given as

$\dot{x}=A x+B_{1} u_{c}+B_{2} v_{T}$

where $u_{c}$ is the continuous time version of switching function $u$ and $v_{T}$ is assumed as load disturbance induced to the converter and neglected here. The discrete-time equivalent of (8) is

$x(k+1)=F x(k)+G_{1} u_{c}(k)+G_{2} v_{T}(k)$

In (9), $u_{c}(k)$ is computed using a suitable state feedback control law. For this, the switching control laws are given by

$u_{c}(k)=-K\left[x(k)-x_{r e f}(k)\right]$

where $K$ is a gain matrix. The gain matrix is obtained from Linear Quadratic Regulator (LQR) method which ensures the desired results for the system while the variations of system load and source parameters are within acceptable limits of reality. From $u_{c}(k)$, the switching function is then generated based on the error level as

$\begin{array}{lll}\text { If } & u_{c}>+h \text { then } & u=+1 \\ \text { elseif } & u_{c}<-h \text { then } & u=-1\end{array}$

where $h$ shows the error level and has a very small positive value. More detail on converter control is given in [16].

\subsection{Resynchronization}

After the fault is cleared in the grid, it is possible to reconnect the MG to grid. Resynchronisation should be carried out when the grid and MG have both same voltage magnitudes and frequencies. Out-of-phase reconnection will cause high currents which can damage the network assets and the network sensitive loads.

Resynchronization of the autonomous MG to grid is usually achieved by detecting the difference in the voltage angle between the MG and grid. In this paper, for resynchronisation, the voltage angle is measured in both sides of the circuit breaker through Phase-Locked Loop (PLL). The circuit breaker closes once the voltage angle is the same on both sides. This prevents huge current and power peaks at resynchronisation period.

As described before, the DERs are running in 3-parameter control mode during autonomous mode. The described resynchronisation can be slow in this case. For a faster resynchronisation, the DER control mode is changed to 2-parameter control mode once the circuit breaker closing is intended.

\subsection{DSTATCOM}

Based on the DER converter control described above, there is no direct voltage control in the MG in autonomous mode. This can be achieved if one of the DERs in MG regulates the network voltage. However, the DERs in residential networks can be owned by customers and are not responsible for network voltage support. Utilizing the converter of a DER to generate reactive power to support the network voltage profile will reduce the active power generation capacity of the converter. This will not be desired by their owners.

Alternatively, voltage regulation in a MG can be achieved using a DSTATCOM [16]. DSTATCOMs have the same converter structure as the DERs. The DSTATCOM can be connected at the secondary side of the distribution transformer to regulate its PCC voltage to a desired value. This can be carried out if a DSTATCOM exchanges reactive power with the network. The reactive power exchange can be controlled using the difference between the PCC RMS voltage and a desired value $\left(V_{T-D S T A T \text {,desired }}\right)$. This difference is utilized to generate the required voltage magnitude across AC filter capacitor in DSTATCOM as

$\left|V_{c f-D S T A T}\right|=V_{c f-D S T A T, r e f}+\left(K_{P}+\frac{K_{I}}{s}\right)\left(V_{T-D S T A T, \text { desired }}-V_{T-D S T A T}\right)$

where $V_{c f-D S T A T, r e f}$ is the assumed reference value for this voltage, $K_{P}$ and $K_{I}$ are PI controller parameters and the suffix DSTAT represents DSTATCOM.

On the other hand, the DC capacitor voltage $\left(V_{d c}\right)$ in DERs is fixed by their DC sources; however, there is no such a DC source in DSTATCOMs. $V_{d c}$ in DSTATCOM can be kept 
equal to its reference value $\left(V_{d c, r e f}\right)$ when the $\mathrm{AC}$ system does not exchange any power with the DC capacitor [17]. This can be reassured if the AC system replenishes the converter losses. For this, the angle of the voltage across the AC filter capacitor $\left(\delta_{c f}\right)$ must be varied versus the DC capacitor voltage variations as

$\delta_{c f-D S T A T, r e f}=\left(K_{P}^{\prime}+\frac{K_{I}^{\prime}}{s}\right)\left(V_{d c-D S T A T, r e f}-V_{d c-D S T A T}\right)$

\section{STUDY CASE AND SIMULATION RESULTS}

For investigating the performance of a network, with selfhealing capability, which includes several MGs different simulation cases are considered. The network performance is investigated in various operating conditions, with different control algorithms for DERs and DSTATCOMs. Some of the simulation cases are discussed below while the network parameters are given in the Appendix.

\subsection{Case 1: Grid-Connected and Autonomous Mode}

Let us consider the simple structure of Fig. 1 to investigate the MG operation during grid-connected and autonomous modes. In grid-connected mode, each DER will generate its rated power and the extra load demand will be supplied by the grid or the extra generation will flow back into the grid. In autonomous mode, total power demand is shared among the DERs proportional to their rating.

The operation of only one MG will be investigated in this section. For this, let us assume in the system of Fig. $1, \mathrm{CB}_{\mathrm{G}}$ and $\mathrm{CB}_{\mathrm{M} 1}$ are closed while $\mathrm{CB}_{\mathrm{M} 2}, \mathrm{CB}_{\mathrm{S} 1}$ and $\mathrm{CB}_{\mathrm{S} 2}$ are open. The system is assumed to be in steady state condition at $t=0$ $\mathrm{s}$ and all the DERs are running in their rated condition.

First, let us assume a 2-parameter control mode is deployed in DERs when the MG is in grid-connected operation which changes to 3-parameter control mode during autonomous operation. At $t=1 \mathrm{~s}$, the grid is disconnected (i.e. $\mathrm{CB}_{\mathrm{G}}$ is opened) and MG-1 will work in autonomous mode. Therefore, the DERs have to increase their output power to satisfy the load demand within the MG. At $t=2 \mathrm{~s}$ a load increase of $3 \mathrm{~kW}$ and at $t=3 \mathrm{~s}$ a load decrease of $3 \mathrm{~kW}$ are applied in the MG. It can be seen that all DERs are sharing the load change proportional to their ratings. At $t=4 \mathrm{~s}$, it is desired to connect the grid. To prevent a huge peak in the current and power, the resynchronization method described before is used. The resynchronization is achieved at $t=4.4 \mathrm{~s}$ and at this time $\mathrm{CB}_{\mathrm{G}}$ is closed and the network is connected back. It can be seen the system reaches to the steady state condition after each change within $0.5 \mathrm{~s}$. Fig. 3(a) shows the active power dispatch of grid and 3 existing DERs in MG-1 between 0 and 5 seconds in the above-mentioned network.

The voltage profile of the network is also shown in Fig. 3(b). As it can be seen in this figure, the network voltage is successfully regulated to $1 \mathrm{pu}$ at all times. The active and reactive power output of the DSTATCOM is also shown in Fig. 3(c). (a)

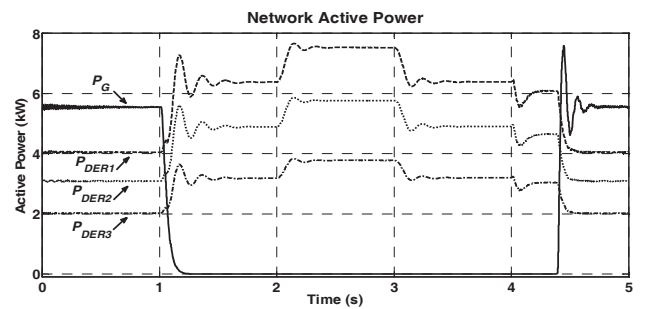

(b)
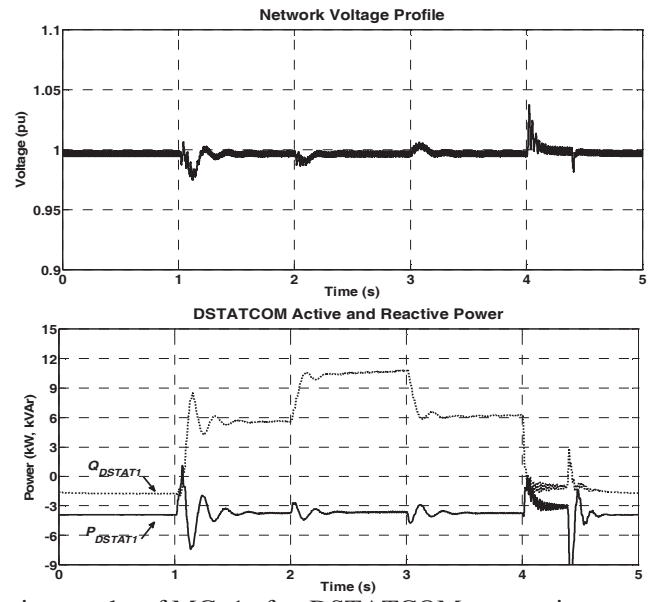

Fig. 3. Simulation results of MG-1 after DSTATCOM connection:

(a) Active power dispatch of grid and 3 DERs,

(b) Network voltage profile in secondary side of distribution transformer,

(c) DSTATCOM output active and reactive injection.

\subsection{Case 2: Interconnected Microgrids}

In this case, let us assume that in network of Fig. 1, at $t=0$ $\mathrm{s}$, circuit breakers $\mathrm{CB}_{\mathrm{G}}, \mathrm{CB}_{\mathrm{M} 1}$ and $\mathrm{CB}_{\mathrm{M} 2}$ are closed and the network is at steady state condition. The considered system has self-healing capability. Now, let us also assume, due to a fault in the MV grid and after self-healing process at $t=1$, $\mathrm{CB}_{\mathrm{G}}$ is open while $\mathrm{CB}_{\mathrm{M} 1}$ and $\mathrm{CB}_{\mathrm{M} 2}$ are closed. As mentioned before, the operation of circuit breakers and resynchronization of interconnecting MGs during self-healing process is not the scope of this research. In this paper, this transition period is not considered and the network performance (i.e. power sharing and voltage regulation) is analyzed after the faulted section is isolated. At $t=2 \mathrm{~s}$ a load increase of $3 \mathrm{~kW}$ and at $t=3 \mathrm{~s}$ a load decrease of $3 \mathrm{~kW}$ are applied in MG-1.

The total active power supply from the grid in addition to the active power flow into each MG feeder is shown in Fig. 4(a). From this figure, it can be seen that MG-2 has a negative power flow and is delivering, approximately, $20 \%$ of the load demand in MG-1. The active power output of all the DERs in MG-1 and MG-2 are shown separately in figures 4(b) and (c), respectively. It can be seen that all DERs in MG-1 and MG-2 are sharing the load demand proportional to their ratings. The voltage profile in the secondary side of the distribution transformers in each MG is regulated to the desired value of 1 pu as shown in Fig. 4(d) and (e).

Now, if there is insufficient generation in MG-1 even when DER are operating at their full capacities while MG-2 has spinning capacity, the extra load demand of MG-1 can be picked up by the DERs in MG-2 and a negative power flow is seen from MG-2 to MG-1. This will prevent or reduce load shedding in MG-1. 


\section{CONCLUSION}

The power management and control of DERs were discussed in this paper, for autonomous operation of the multiple interconnected microgrids, in a medium voltage feeder with self-healing capability. The microgrids consisted of several converter-interfaced micro sources where a decentralized power sharing algorithm based on droop control was used. Different control algorithms were developed to manage the DERs at grid-connected and autonomous operation modes. Based on the described control method, a DER with extra generation capacity can share some of the loads in other microgrids, once the other microgrids have a shortage of generation. This can prevent or reduce the load shedding in some of the interconnected microgrids. The network voltage is regulated using the DSTATCOMs installed in each microgrid.

\section{APPENDIX}

Table I. Technical data of the network parameters of Fig. 1.

\begin{tabular}{ll}
\hline MV Network & $11 \mathrm{kV} \mathrm{L}-\mathrm{L}$ RMS, $50 \mathrm{~Hz}$ \\
MV Line Impedances & $R=0.2 \Omega, L=10 \mathrm{mH}$ \\
LV Feeder & $410 \mathrm{~V} \mathrm{~L}-\mathrm{L}$ RMS, $50 \mathrm{~Hz}$ \\
LV Line Impedances & $R=0.02 \Omega, L=1 \mathrm{mH}$ \\
Transformers & $30 \mathrm{kVA}, 11 \mathrm{kV} / 410 \mathrm{~V}$, Three-Phase, $\Delta /$ \\
& Y-Grounded, $Z_{I}=5 \%$, \\
Impedance Loads & 7 three-phase RL load each $P=3 \mathrm{~kW}, P F$ \\
& $=0.95$ \\
Induction Motors & 2 three-phase each $P=1.5 \mathrm{~kW}, P F=0.8$ \\
DER VSCs and Filters & $R_{f}=0.1 \Omega, L_{f}=4.1 \mathrm{mH}, C_{f}=50 \mu \mathrm{F}, V_{d c}=$ \\
& $150 \mathrm{~V}, a=3.33, h=10^{-5}$ \\
DSTATCOM VSCs and Filters & $R_{f}=0.1 \Omega, L_{f}=4 \mathrm{mH}, C_{f}=25 \mu \mathrm{F}, V_{d c}=1$ \\
& $\mathrm{kV}, a=1, h=10^{-5}$ \\
\hline
\end{tabular}

Table II. Technical data of the DERs and droop control coefficients in Fig. 1.

\begin{tabular}{lcclc}
\hline DER Type & $\begin{array}{l}\text { DER Rating } \\
{[\mathrm{kW}]}\end{array}$ & $\begin{array}{l}\text { Inductance }\left(L_{T}\right) \\
{[\mathrm{mH}]}\end{array}$ & $\begin{array}{l}m \\
{[\mathrm{rad} / \mathrm{kW}]}\end{array}$ & $\begin{array}{c}n \\
{[\mathrm{kV} / \mathrm{kVAr}]}\end{array}$ \\
DER-1,4 & 4 & 38.1 & 225 & 7.68 \\
DER-2,5 & 3 & 49.8 & 400 & 10.25 \\
DER-3 & 2 & 76.2 & 450 & 15.37 \\
\hline
\end{tabular}

REFERENCES

[1] X. Fang, S. Misra, G. Xue, and D. Yang, "Smart grid- The new and improved power grid: A survey," IEEE Communications Surveys \& Tutorials, Vol. 14, No. 4, pp. 944-980, 2012.

[2] K. Moslehi and R. Kumar, "A reliability perspective of the smart grid," IEEE Trans. on Smart Grid, Vol. 1, No. 1, pp. 57-64, 2010.

[3] H. Liu, X. Chen, K. Yu and Y. Hou, "The control and analysis of selfhealing urban power grid,", IEEE Trans. on Smart Grid, Vol. 3, No. 3, pp. 1119-1129, Sep. 2012.

[4] N. Hatziargyriou, H. Asano, R. Iravani and C. Marnay, "Microgrids," IEEE Power and Energy Magazine, Vol. 5, No. 4, pp. 78-94, 2007.

[5] J.A.P. Lopes, C.L. Moreira and A.G. Madureira, "Defining control strategies for microgrids islanded operation," IEEE Trans. on Power Systems, Vol. 21, No. 2, pp. 916-924, 2006.

[6] S. Rahman, M. Pipattanasomporn and Y. Teklu, "Intelligent distributed autonomous power systems (IDAPS)," IEEE Power Engineering Society General Meeting, pp. 1-8, June 2007.

[7] R.J. Yinger, "Self-healing circuits at Southern California Edison," IEEE Transmission and Distribution Conf. pp. 1-3, May 2012.

[8] M. Amin, "Toward a self-healing energy infrastructure," IEEE Power Engineering Society General Meeting, pp. 1-7, 2006.

[9] K. Moslehi, A.B.R. Kumar and P. Hirsch, "Feasibility of a selfhealing grid- Part II: Benefit models and analysis," IEEE Power Engineering Society General Meeting, pp. 1-8, 2006.

[10] K. Moslehi, A.B.R. Kumar, D. Shurtleff, M. Laufenberg, A. Bose and P. Hirsch, "Framework for a self-healing power grid," IEEE Power Engineering Society General Meeting, pp. 1-8, June 2005. (a)

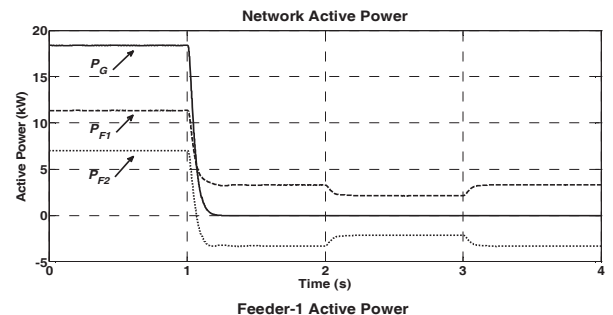

(b)
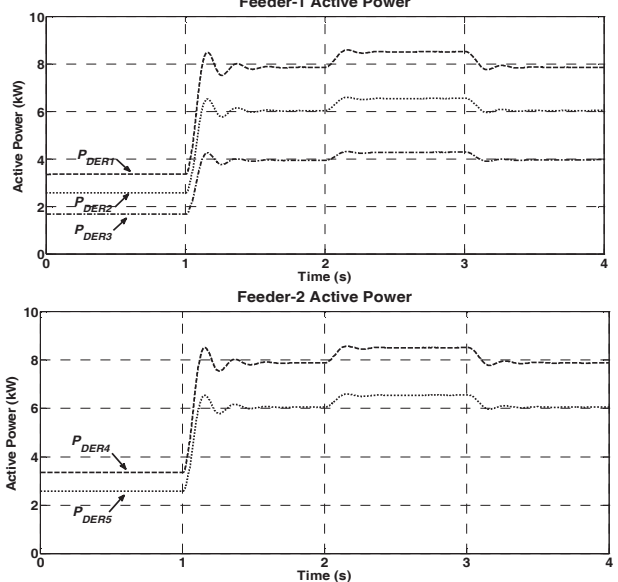

Feeder-1 Voltage Profile
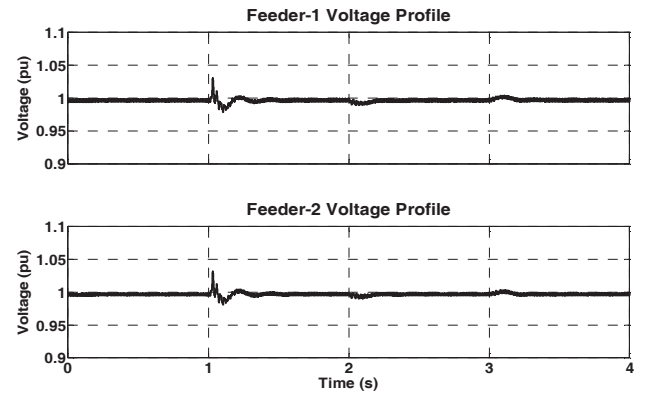

Fig. 4. Simulation results of interconnected MG-1 and MG-2:

(a) Active power supply from grid and active power flow into each MG,

(b) Active power output of each DER in MG-1,

(c) Active power output of each DER in MG-2,

(d) MG-1 voltage profile in secondary side of distribution transformer,

(e) MG-2 voltage profile in secondary side of distribution transformer.

[11] C.M. Colson, M.H. Nehrir and R.W. Gunderson, "Distributed multiagent microgrids: A decentralized approach to resilient power system self-healing," IEEE $4^{\text {th }}$ Int. Symp. on Resilient Control Systems (ISRCS), pp. 83-88, Aug. 2011.

[12] D. Li, S. Wang, J. Zhan and Y. Zhao, "A self-healing reconfiguration technique for smart distribution networks with DGs," IEEE Int. Conf. on Electrical and Control Engineering, pp. 4318-4321, Sept. 2011.

[13] A. Košt'álová and P.M.S. Carvalho, "Towards self-healing in distribution networks operation: Bipartite graph modelling for automated switching," Electric Power Systems Research, Vol. 81, Issue 1, pp. 51-56, Jan. 2011.

[14] Q. Pang, H. Gao and M. Xiang, "Design of intelligent terminal unit for smart distribution grid," IEEE China Int. Conf. on Electricity Distribution (CICED), pp.1-6, Sept. 2010.

[15] F. Shahnia, R. Majumder, A. Ghosh, G. Ledwich and F. Zare, "Operation and control of a hybrid microgrid containing unbalanced and nonlinear loads," Electric Power Systems Research, Vol. 80, Issue 8, pp. 954-965, August 2010.

[16] A. Ghosh, G. Ledwich, Power quality enhancement using custom power devices, Kluwer Academic Publishers, 2002.

[17] A. Ghosh and G. Ledwich, "Load compensating DSTATCOM in weak AC systems," IEEE Trans. on Power Delivery, Vol. 18, No. 4, pp. 1302-1309, Oct. 2003. 